tein-1 (SREBF1) and localization of SREBF1 and SREBF2 to chromosomes $17 \mathrm{p} 11.2$ and $22 \mathrm{q} 13$. Genomics. 25:667-673.

25. Ravinet Trillou, C., et al. 2003. Anti-obesity effect of SR141716, a CB1 receptor antagonist, in dietinduced obese mice. Am. J. Physiol. Regul. Integr. Comp. Physiol. 284:R345-R353.
26. Poirier, B., et al. 2005. The anti-obesity effect of rimonabant is associated with an improved serum lipid profile. Diabetes Obes. Metab. 7:65-72.

27. Cravatt, B.F., et al. 2001. Supersensitivity to anandamide and enhanced endogenous cannabinoid signaling in mice lacking fatty acid amide hydrolase. Proc. Natl. Acad. Sci. U. S. A. 98:9371-9376.
28. Hillard, C.J., and Campbell, W.B. 1997. Biochemistry and pharmacology of arachidonylethanolamide, a putative endogenous cannabinoid. J. Lipid Res. 38:2383-2398.

29. Fride, E., et al. 2001. Critical role of the endogenous cannabinoid system in mouse pup suckling and growth. Eur. J. Pharmacol. 419:207-214.

\title{
Chemokine-mediated angiogenesis: an essential link in the evolution of airway fibrosis?
}

\author{
Ivor S. Douglas ${ }^{1,2}$ and Mark R. Nicolls ${ }^{1}$ \\ 1Department of Medicine, Pulmonary Sciences and Critical Care Medicine and 2 Denver Health Medical Center, \\ University of Colorado Health Sciences Center, Denver, Colorado, USA.
}

\begin{abstract}
Angiogenesis may be an important factor in the development of fibrotic lung disease. Prior studies have strongly suggested a role for angiogenic vascular remodeling in pulmonary fibrosis, and emerging evidence indicates that new vessel formation is critical in airway fibrosis. Bronchiolitis obliterans syndrome is a fibrotic occlusion of distal airways that is largely responsible for the morbidity and mortality of patients after lung transplantation. In this issue, Belperio et al. demonstrate a role for CXC chemokine receptor 2 in the regulation of angiogenesis-mediated airway fibroproliferation (see the related article beginning on page 1150). By integrating an understanding of neovascularization into the study of events that occur between inflammation and fibrosis, it becomes increasingly possible to rationally design therapies that can halt conditions of maladaptive fibrosis.
\end{abstract}

Neovascularization is an important component of fibrotic responses (1). In this issue of the JCI, Belperio and colleagues extend this relationship to the development of chronic lung transplant rejection (2). Using bronchoalveolar lavage fluid from patients with pending or established bronchiolitis obliterans syndrome (BOS) and tracheal allograft tissue from a mouse model of obliterative airway disease, the authors make a convincing case for the central role of CXC chemokine receptor 2 (CXCR2) regulation of angiogenesis-mediated airway fibroproliferation.

\section{Airway inflammation and fibrosis in the evolution of BOS}

Chronic allograft rejection is the chief factor limiting long-term survival following lung transplantation. BOS is the pathological correlate of chronic rejection and primarily

Nonstandard abbreviations used: BOS, bronchiolitis obliterans syndrome; CXCL, CXC chemokine ligand; CXCR2, CXC chemokine receptor 2.

Conflict of interest: The authors have declared that no conflict of interest exists.

Citation for this article: J. Clin. Invest. 115:1133-1136 (2005). doi:10.1172/JCI200525193. affects the respiratory and terminal bronchioles, which culminates in a fibrotic occlusion of the distal airways (3). The cumulative incidence of BOS at 5 years after lung transplant is between $50 \%$ and $80 \%$, and 5-year survival after BOS onset is only $30-50 \%$. The International Society of Heart and Lung Transplantation Registry has noted that the development of BOS within the first year after transplantation is the single most important factor influencing 5-year mortality among patients undergoing lung transplantation (3). As a fibrotic disease, BOS is poorly responsive to standard immunosuppression employed by transplant physicians. Similarly, pulmonary fibrosis, which affects the lung interstitium rather than the conducting airways, responds poorly to immunotherapy and has long been associated with pathologic angiogenesis (4).

It is a generally recognized phenomenon that inflammation is an initiating event that precedes the progression to fibrosis in several lung diseases, including BOS and idiopathic pulmonary fibrosis. While fibrosis may be a frequent sequel of an acute or subacute inflammatory event, it is also clear that inflammation does not always result in fibrosis. The long-term effect of interstitial or airway fibrosis is irreversible lung architectural remodeling. Key questions regarding the mechanisms of airway remodeling are: (a) What are the specific inflammatory initiators? and (b) What is the sequence of events that culminates in fibroproliferation? In lung transplantation, the answer to the first question most certainly involves the response to alloantigen triggering of innate and adaptive immune responses. The answer to the second question is probably less well understood but is perhaps of greater importance in the development of therapies that reach beyond immunosuppression. Lung transplant clinicians well appreciate that acute rejection treated early may respond excellently to immunosuppressive therapies but that late intervention is rarely successful. Unfortunately, it is not always possible to intervene early, and occasionally even apparently early intervention with highdose steroids or T cell-depleting strategies cannot halt the decline in lung function once fibroproliferation is initiated.

\section{The potential role of CXC chemokines in angiofibroproliferative BOS}

The study by Belperio and colleagues (2) firmly establishes that neovascularization is an important contributor to the process of fibroproliferation in airway fibrosis. The investigators present a cohesive and clearly argued interpretation of experimental data from human BOS patients and a well-characterized murine model of tracheal transplant rejection. Their findings make a convincing case for the central role of CXCR2-dependent Glu-LeuArg-positive $\left(\mathrm{ELR}^{+}\right)$chemokine regulation of angiogenesis-mediated BOS fibroproliferation. The study extends their previ- 
commentaries

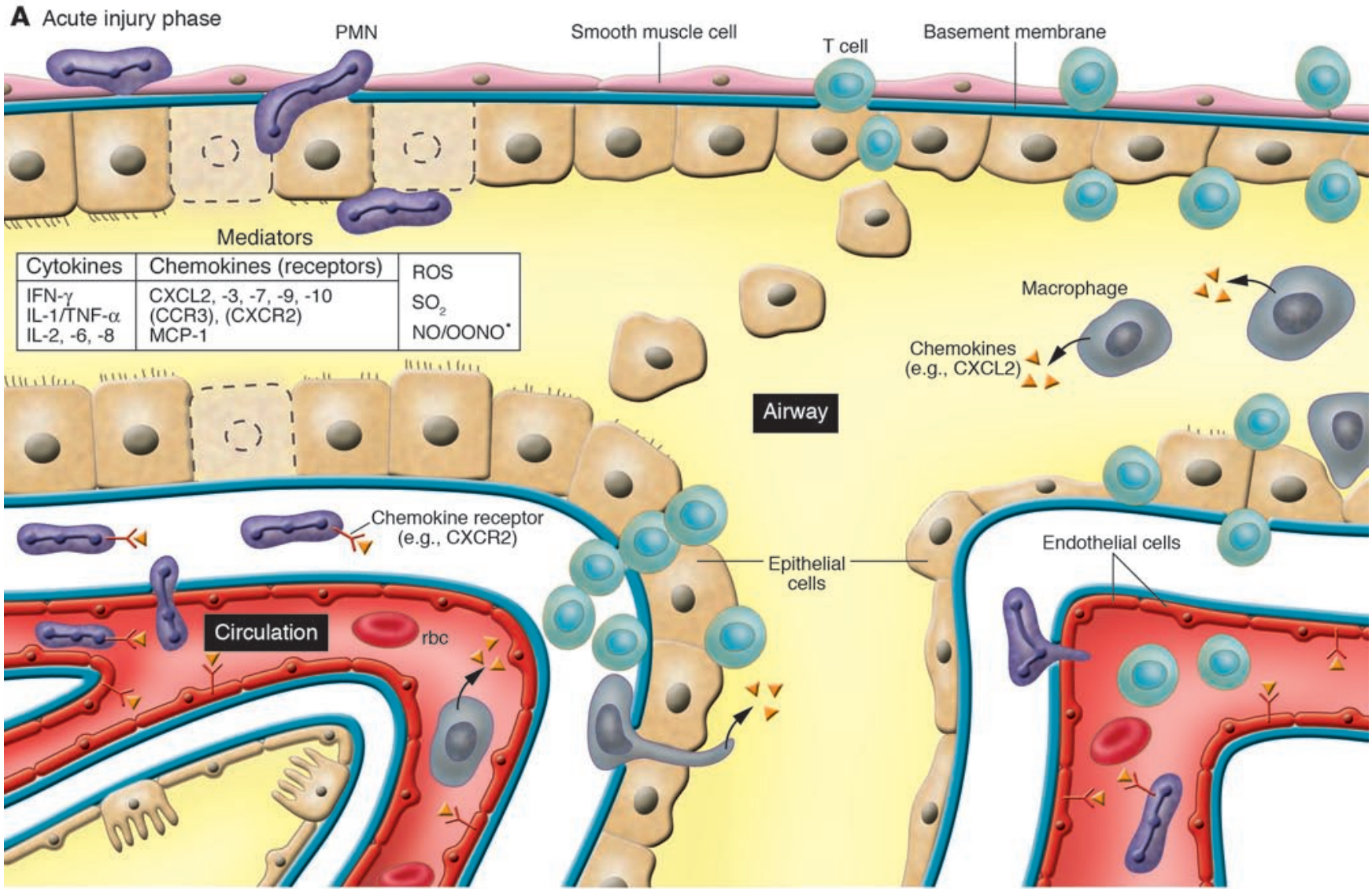

B Angio-fibroproliferative phase
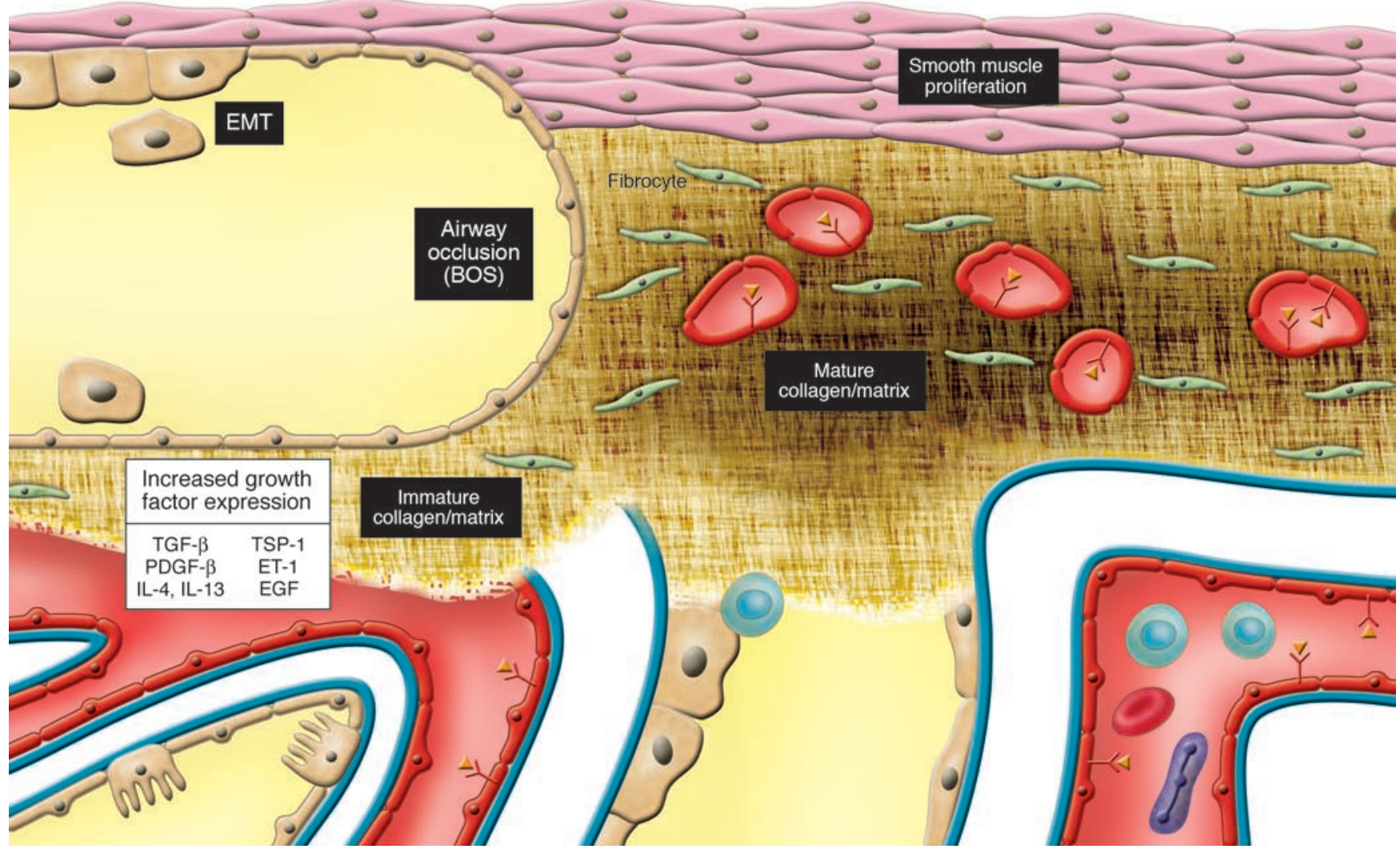
ously published observations based on the BOS model, which implicated monocyte chemoattractant protein-1/CXCR2-mediated mononuclear phagocytic infiltration in the progression of BOS (5). CXC chemokines, 1 of the 4 subfamilies of multifunctional growth- and immune-mediating molecules, regulate acute inflammatory responses and vascular remodeling. CXC chemokines contain an $\mathrm{ELR}^{+}$motif immediately before the first cysteine residue at the $\mathrm{NH}_{2}$ terminus. This group includes CXC ligand 1 (CXCL1), -2, -3, -5, -6, and -8. Angiogenesis (both physiological and pathological) is potently stimulated by engagement of these ligands with $G$ proteincoupled CXCR2 on endothelial cells. By contrast, IFN-regulated ELR ${ }^{-}$chemokines such as CXCL4, $-9,-10$, and -11 function as angiostatic regulators through CXCR3 and direct interaction with angiogenic factors such as VEGF (6).

The elucidation of VEGF-independent, chemokine-mediated angiogenesis is pivotal to our evolving understanding of the cascade of remodeling events that results in inexorable progression of fibroproliferative BOS. The present study also demonstrates a temporal separation of signaling responses to CXCR2 activation. CXCR2 engagement in early murine tracheal allograft BOS results in neutrophil trafficking, but at 3 weeks results in neutrophil-independent angiofibroproliferation (2). Moreover, treatment of CXCR2 $2^{-/-}$mice with threshold doses of cyclosporin, insufficient to prevent BOS in wild-type mice but sufficient to inhibit early monocyte infiltration, resulted in a dramatic reduction in allograft fibroobliteration, which points to a clinically relevant strategy for further investigation.

How does pathological angiogenesis fit into what is already known or generally posited for lung fibrosis? Briefly, there is evi- dence to suggest that the epithelium can be an early target as well as a source of alloantigen (7). Following exposure to a new antigen or toxin, there is an influx of neutrophils and mononuclear cells (Figure 1A). This period of cellular infiltration is associated with an increased production of chemokines and cytokines. In this cascade, multiple changes occur in the epithelia, including apoptosis, hyperplasia, dedifferentiation, and metaplasia (8-12). Epithelial changes may stimulate underlying myofibroblasts in a paracrine fashion, with resultant collagen matrix deposition (13-15). In the midst of these changes, neovascularization is observed (Figure 1B). Perhaps as a consequence of increased vascularity and chemoattractant gradients, migratory cell populations (e.g., fibrocytes; ref. 16) traffic to the site of inflammation, possibly as part of a tissue repair response. As this process progresses, the cellular infiltrates diminish $(8,9)$, and airway remodeling proceeds in a unidirectional fashion, with extensive subepithelial, luminal, and/or interstitial fibrosis. Our group has recently established using an orthotopic (in situ) mouse tracheal transplant model that it is possible to reverse alloimmune injury that remains unmitigated for 7 days, but after 10 days of unprotected immune airway injury, fibroproliferative diseases cannot be ameliorated (8). Thus, it appears that a temporal sequence of events leading from inflammation to fibrosis may be closer to being fully elucidated.

In addition to alloimmune injury, several important potential amplifiers of BOS have been identified. These include chronic gastroesophageal reflux (17), communityacquired viral infection (18), and CMV infection (19), all of which are also associated with chronic graft rejection. Chemokinemediated inflammation and angiogenesis may be a common pathway for progression to fibroproliferative BOS in response to superimposition of these factors on an immune-injured airway. For example, virulent CMV strains produce a potent ELR ${ }^{+}$ chemokine analog, UL146 (also known as viral CXCL1). This CXCR2-restricted ligand is capable of inducing neutrophil chemotaxis independent of monocyte activation (20) and could potentially contribute to chemokine-mediated perigraft angiogenesis and fibroproliferation.

Belperio and colleagues' important observations (2) hold great promise for translational application in the therapy of BOS and other fibrotic lung diseases that involve pathological angiofibroproliferation. Presently, prevention of graft rejection requires intensive, prolonged immunosuppression with corticosteroids, calcineurin antagonists, antimetabolites, and immune modulators (21). This approach, which is focused on the inflammatory component, has clear limitations, with significantly increased risks of opportunistic infection and a limited effect on angiogenesis. An obvious therapeutic strategy would be treatment with a combination of lower doses of traditional immunosuppressants and humanized blocking antibodies against angiogenic chemokines or their receptors, as modeled in the present study (2). If this broad framework holds true for most fibrotic conditions, in the future, it may be possible to more appropriately treat other pulmonary, renal, dermatological, and cardiac diseases in which the inflammation-angiogenesis-fibroproliferative pathways are active.

Address correspondence to: Ivor S. Douglas, University of Colorado Health Sciences Center, 4200 East 9th Avenue, School of Medicine 5226, Mail stop C-272, Denver, Colorado 80262, USA. Phone: (303) 436-5905; Fax: (303) 436-7249; E-mail: idouglas@dhha.org.

\section{Figure 1}

Evolution of BOS from airway injury to angiofibroproliferation. (A) The progression of airway pathology from initial injury to luminal and subepithelial fibrosis begins with cellular infiltration consisting of macrophages (a significant source of CXCL2), neutrophils, and subsequently T lymphocytes. Infiltrating cells and injured lung parenchyma (e.g., epithelial cells, vascular endothelial cells) liberate a variety of cytokines, oxidants, and chemokines. One prominently expressed chemokine, CXCL2, binds to receptors on neutrophils and vascular endothelium, simultaneously promoting a neutrophil influx and angiogenesis. Following cellular infiltration, the respiratory epithelium is likely injured, and pseudostratified columnar epithelium undergoes apoptosis and is transformed into a flattened, dysplastic monolayer that covers the basement membrane. (B) During a period when the epithelium is undergoing progressive injury, angiogenesis likely occurs through a CXCR2-dependent, VEGF-independent process (2). While the mechanism by which angiogenesis contributes to progressive airway fibrosis has not yet been fully elucidated, it is possible that increased vascularity facilitates the delivery of growth factors that promote tissue remodeling (e.g., platelet-derived growth factor-2 [PDGF2]) or cells that differentiate to produce collagen (e.g., fibrocytes). The concurrent and persistent immune epithelial injury may result in epithelial-myofibroblast crosstalk that promotes new collagen synthesis (22) through paracrine growth factor signaling and epithelial-to-mesenchymal transition (EMT). As subepithelial fibrosis increases and the respiratory epithelium becomes less distinct, increased deposition in the airway lumen of type I and III collagen occurs. Eventually, this fibrosis culminates as BOS, a physiologically significant occlusion of the airways in lung transplant recipients, which is the hallmark of chronic lung transplant rejection. PMN, polymorphonuclear neutrophil; ET-1, endothelin-1; EGF, epidermal growth factor. 
1. Kalluri, R., and Sukhatme, V.P. 2000. Fibrosis and angiogenesis. Curr. Opin. Nephrol. Hypertens. 9:413-418.

2. Belperio, J., et al. 2005. Role of CXCR2/CXCR2 ligands in vascular remodeling during bronchiolitis obliterans syndrome. J. Clin. Invest. 115:1150-1162. doi:10.1172/JCI200524233.

3. Estenne, M., and Hertz, M.I. 2002. Bronchiolitis obliterans after human lung transplantation. Am J. Respir. Crit. Care Med. 166:440-444.

4. Turner-Warwick, M. 1963. Precapillary systemicpulmonary anastomoses. Thorax. 18:225-237.

5. Belperio, J.A., et al. 2001. Critical role for the chemokine MCP-1/CCR2 in the pathogenesis of bronchiolitis obliterans syndrome. J. Clin. Invest. 108:547-556. doi:10.1172/JCI200112214.

6. Belperio, J.A., et al. 2000. CXC chemokines in angiogenesis. J. Leukoc. Biol. 68:1-8.

7. Fernandez, F.G., et al. 2004. Airway epithelium is the primary target of allograft rejection in murine obliterative airway disease. Am. J. Transplant. 4:319-325.

8. Murakawa, T., et al. 2005. Simultaneous LFA-1 and CD40 ligand antagonism prevents airway remodeling in orthotopic airway transplantation: implications for the role of respiratory epithelium as a modulator of fibrosis. J. Immunol. 174:3869-3879.

9. Minamoto, K., and Pinsky, D.J. 2002. Recipient iNOS but not eNOS deficiency reduces luminal narrowing in tracheal allografts. J. Exp. Med. 196:1321-1333.

10. Neuringer, I.P., et al. 2002. Epithelial kinetics in mouse heterotopic tracheal allografts. Am. J. Transplant. 2:410-419.

11. Reader, J.R., et al. 2003. Pathogenesis of mucous cell metaplasia in a murine asthma model. Am. J. Pathol. 162:2069-2078.

12. Kasper, M., and Haroske, G. 1996. Alterations in the alveolar epithelium after injury leading to pulmonary fibrosis. Histol. Histopathol. 11:463-483.

13. Howat, W.J., Holgate, S.T., and Lackie, P.M. 2002. TGF-beta isoform release and activation during in vitro bronchial epithelial wound repair. Am.J. Physiol. Lung Cell Mol. Physiol. 282:L115-L123.

14. Morishima, Y., et al. 2001. Triggering the induction of myofibroblast and fibrogenesis by airway epithelial shedding. Am. J. Respir. Cell Mol. Biol. 24:1-11.

15. Zhang, S., Smartt, H., Holgate, S.T., and Roche, W.R. 1999. Growth factors secreted by bronchial epithelial cells control myofibroblast proliferation: an in vitro co-culture model of airway remodeling in asthma. Lab. Invest. 79:395-405.

16. Phillips, R.J., et al. 2004. Circulating fibrocytes traffic to the lungs in response to CXCL12 and mediate fibrosis. J. Clin. Invest. 114:438-446. doi:10.1172/ JCI200420997.

17. Palmer, S.M., et al. 2000. Gastroesophageal reflux as a reversible cause of allograft dysfunction after lung transplantation. Chest. 118:1214-1217.

18. Chakinala, M.M., and Walter, M.J. 2004. Community acquired respiratory viral infections after lung transplantation: clinical features and longterm consequences. Semin. Thorac. Cardiovasc. Surg. 16:342-349.

19. Zamora, M.R. 2004. Cytomegalovirus and lung transplantation. Am. J. Transplant. 4:1219-1226.

20. Penfold, M.E., et al. 1999. Cytomegalovirus encodes a potent alpha chemokine. Proc. Natl. Acad. Sci.U. S. A. 96:9839-9844.

21. Knoop, C., Haverich, A., and Fischer, S. 2004. Immunosuppressive therapy after human lung transplantation. Eur. Respir. J. 23:159-171.

22. Selman, M., and Pardo, A. 2002. Idiopathic pulmonary fibrosis: an epithelial/fibroblastic cross-talk disorder. Respir. Res. [serial online]. 3:3. http://respiratory-research.com/content $/ 3 / 1 / 3$.

\title{
The role of hepatic insulin receptors in the regulation of glucose production
}

\author{
Alan D. Cherrington
}

Department of Molecular Physiology and Biophysics, Vanderbilt University School of Medicine, Nashville, Tennessee, USA.

\begin{abstract}
The inability of insulin to suppress hepatic glucose production (HGP) is a key defect found in type 2 diabetes. Insulin inhibits HGP through both direct and indirect means, the latter of which include inhibition of glucagon secretion, reduction in plasma nonesterified fatty acid level, decrease in the load of gluconeogenic substrates reaching the liver, and change in neural signaling to the liver. Two studies in this issue of the JCI demonstrate that selective changes in the expression of insulin receptors in mouse liver do not have a detectable effect on the ability of insulin to inhibit HGP (see the related articles beginning on pages 1306 and 1314). These provocative data suggest that the indirect effects of insulin on the liver are the primary determinant of HGP in mice.
\end{abstract}

\section{Introduction}

Until late 1987, it was believed that insulin's ability to reduce hepatic glucose production (HGP) resulted from the direct interaction of the hormone with its receptor in the hepatocyte plasma membrane. This belief was called into question when Prager et al. (1) noted that in obese, nondiabetic humans, suppression of glucose production could occur in response to insulin

Nonstandard abbreviations used: HGP, hepatic glucose production; LIRKO, liver insulin receptor knockout; NEFA: nonesterified fatty acid; NHGO, net hepatic glucose output.

Conflict of interest: The author has declared that no conflict of interest exists.

Citation for this article: J. Clin. Invest. 115:1136-1139 (2005). doi:10.1172/JCI200525152 infusion, even when the estimated portal vein insulin concentration did not rise. These results suggested that insulin also reduces hepatic glucose output by indirect mechanisms. Subsequent work by others supported this concept (2-5), and it is now recognized that insulin can inhibit HGP by both direct and indirect means (Figure 1).

Indirect actions of insulin on the liver The indirect actions of insulin on HGP are diverse. Glucagon secretion from the $\alpha$ cell of the pancreas is diminished by insulin, which in turn causes a reduction in HGP (6). Likewise, nonesterified fatty acid (NEFA) release from the adipocyte is reduced by insulin, and a reduction in the supply of NEFAs to the liver causes an increase in hepatic glycolytic flux, resulting in glucose-6-phosphate exiting the liver after being converted to lactate rather than glucose (3). Additionally, the effect of insulin on fat and muscle reduces the supply of gluconeogenic precursors reaching the liver, again reducing $\operatorname{HGP}(2,7)$. More recently, insulin's action in the brain has been postulated to play a role in the regulation of HGP (8). It is known that the brain can sense the circulating insulin level (9) and that it provides neural input to the liver (10). Further, it has been shown that infusion of insulin into the third ventricle of rats can reduce glucose production (8). Likewise, blocking insulin action in the rat hypothalamus impairs the ability of a physiologic rise in circulating insulin to inhibit HGP (8).

\section{Direct actions of insulin on the liver}

The exploration of insulin's indirect effects on the liver called into question the physiologic relevance of the hormone's direct hepatic effect, even though numerous in vitro studies had shown it to exist. Perhaps the best in vivo demonstration of the hormone's direct effect on the liver comes from studies conducted in the conscious overnight-fasted dog, in which changes in plasma insulin were brought about selectively in the liver using the pancreatic clamp technique. 